\title{
Giant radio galaxies as probes of the large-scale structure
}

\section{Lakshmi Saripalli*}

Raman Research Institute, C.V. Raman Avenue, Sadashivanagar, Bangalore, India

E-mail: lsaripalerri.res.in

Jurek M Malarecki

ICRAR, University of Western Australia

Radio galaxies, which are visible symbols of interaction between twin jets and the ambient medium, are well recognized for their potential in probing the gaseous environment. I will describe results from our recent work where we have used a large sample of giant radio galaxies to probe the gaseous medium on large scales using both radio continuum imaging and optical spectroscopy. We find jets and lobes to be sensitive probes of the unseen ambient medium associated with low density galaxy concentrations and filaments. The study has implications for gauging how the WHIM gas is distributed and for the long-standing question of why some radio galaxies grow to giant sizes. The work is discussed in the context of upcoming sensitive radio surveys and instruments.

EXTRA-RADSUR2015 (*)

20-23 October 2015

Bologna, Italy

(*) This conference has been organized with the support of the Ministry of Foreign Affairs and International Cooperation, Directorate General for the Country Promotion (Bilateral Grant Agreement ZA14GR02 - Mapping the Universe on the Pathway to SKA)

\footnotetext{
* Speaker.
} 


\section{Introduction}

The motivation in this presentation is to showcase giant radio galaxies as tools that carry immense potential to study the large-scale galaxy and gas environments. They provide fresh insights and complementary efforts to the traditional means of studying environments on large scales. The work reported at the meeting was based on investigations carried out by Jurek Malarecki for his $\mathrm{PhD}$ thesis (University of Western Australia) for which together with Lister Staveley-Smith, Heath Jones and Ravi Subrahmanyan, the speaker was one of the supervisors. We embarked on this project three years ago and towards this we obtained some exquisite data in both radio and optical bands. The results reported here may be found in Malarecki et al. (2013) and Malarecki et al. (2015).

We know of the Intergalactic medium (IGM) today as a vast cosmic web of gas that strongly evolved over time from a largely neutral state to one in a significantly ionized state. The spectacular transformation in the physical state of the IGM is testimony to its close link with galaxies, which also form within and from it. The IGM, where a substantial fraction of the baryonic matter resides, is by definition in regions well outside of rich groups and clusters of galaxies and outside as well of the circum-galactic or the hot intra-cluster medium associated with galaxies and clusters. The multi-phasic and inhomogeneous IGM contains concentrations of neutral hydrogen of various densities and extents, and gaseous components of different temperatures. This cosmic web of matter is known to be filamentary via galaxy redshift surveys and via numerical simulations that suggest gas flows within the filaments aggregating on to the nodal regions that contain rich galaxy clusters and groups. The inflows of cool gas result in shock heating as the gas encounters deep darkmatter potentials. At more recent cosmic time the IGM begins to show traces of highly ionized metal species.

Much of the information we have of the IGM and its physical nature is via absorption line studies in radio and optical wavelengths and through modeling. While our knowledge of the distant IGM is gleaned from the strong signatures in the spectra of distant quasars, the transformation of its physical state too is well recorded in the quasar optical spectra with a depleting forest of absorption lines short-ward of rest-frame Lyman- $\alpha$ line in going from more distant to nearby quasars. In addition, today sophisticated observations that image and obtain 2-dimensional spectra of faint distributions of the IGM gas are beginning to unveil the IGM gas distribution (in the vicinity of redshift 2-3 quasars) showing its filamentary nature and dynamical state (Cantalupo et al. 2014, Martin et al. 2014 and Martin et al. 2015).

Ironically, at lower redshifts the information we have of the IGM is severely incomplete, with a deficit of as much as 30 percent recognized in the inventory of all baryonic matter contained in galaxies, X-ray halos and Lyman- $\alpha$ clouds (Shull et al. 2012). The puzzle of the "missing baryons", as it came to be referred to, found a solution in the form of a prediction, made as a result of hydrodynamical simulations of galaxy formation, by Cen and Ostriker (1999) where a large amount of infalling and shock-heated gas is expected at lower redshifts at enhanced temperatures of $10^{5}-10^{7} \mathrm{~K}$.

In proximity to galaxy groups and clusters the shock-heated gas is expected to also be enriched by outflows from the galaxies and hence enriched by metals. The various modes of interaction, whether via inflowing gas or outflows or heating and mixing, all suggest a dynamic state for the 
IGM and its close relationship with galaxies and structure formation.

The prediction of the existence of a $10^{5}-10^{7} \mathrm{~K}$ heated ionized gas (the Warm-Hot Intergalactic Medium or the WHIM) led to a spate of detection exercises. However, compounded by the tenuousness caused by expansion of the universe, the high temperatures to which it is heated and the nature of the WHIM gas, it is rendered mostly invisible to traditional means of observing such as Lyman- $\alpha$ absorption features or $21-\mathrm{cm}$ absorption or emission or X-ray emission. While the few and far between denser components of neutral hydrogen are mostly detected in Lyman- $\alpha$ absorption the diffuse neutral component of the local IGM has been observed via HI emission, albeit with great effort (Popping and Braun, 2011). Most of the detection efforts for the WHIM, on the other hand, have been carried out via absorption-line observations of highly ionized metals against bright background quasars.

The search experiments for the WHIM have resulted in several tentative detections. Even so, with the available tool dependent on sparse metal ions and discrete lines of sight towards background quasars, the exercises are handicapped by signal/noise concerns and low statistics. Recently, the WHIM has been pursued via X-ray emission observations but only after stacking 41 cluster observations (Fraser-McKelvie et al 2011).

The difficulty in detecting the WHIM and its distribution as well as its components raises need for other tools to be employed and in this context the use of the largest radio galaxies is a worthy exercise to explore (Malarecki et al, 2013, Malarecki et al, 2015).

\section{Using giant radio galaxies in the study of large-scale structure}

With median linear sizes of radio galaxies typically at $200 \mathrm{kpc}$ (Singal, 1993), the relatively rare population of radio galaxies with linear sizes more than three times larger have come to be described as giant radio galaxies (GRGs) whose lobes extend to and propagate in regions well away from the host galaxy and its associated thermal halo. Figure 1 nicely illustrates the spectacular size differences using the $1 \mathrm{Mpc}$ GRG, B1545-321 (Saripalli et al 2003). This aspect of GRGs, of their lobes extending out to large scales, has been used previously to probe the ambient medium on these scales, the inter-galactic medium (IGM). However, with sophisticated simulations of the WHIM now available and tools to map the large-scale galaxy environments using 2-D spectroscopic surveys, GRGs take on an added significance as probes of ambient gas, giving a ring-side view of the gas and galaxies on large scales.

Towards this goal we had previously carried out two case studies using GRGs B0503-286 and B0319-456, both distinctly asymmetric in several respects (whether in lobe extents or their morphology or fluxes; Subrahmanyan et al, 2008 and Safouris et al, 2009). Both the exercises, revealed a clear and presumably causative relationship between the large-scale galaxy environment (mapped by the 2-degree Field survey) and the radio properties of the two GRGs. The lobes were found to extend to a shorter extent on the side that has a higher concentration of galaxies, with the lobes also showing signs of being displaced into regions away from regions of higher galaxy densities. These early case studies gave rise to an ambitious investigation of a much larger sample of 19 southern giant radio galaxies (linear sizes, $0.8-3.2 \mathrm{Mpc}, \mathrm{z}<0.15$ ).

An expectation of using GRGs for IGM studies is that if their lobes (presumably old and relaxed) trace regions that are in near pressure balance with the ambient medium, their lobe pressure 
estimates (obtained using minimum energy arguments) will directly reflect on the ambient medium in which they are located. Combined with galaxy distribution in the vicinity of the lobes gas-galaxy relationship can be traced hence paving the way for more detailed studies of the WHIM. Unlikely to be a homogeneous, single-component medium, the WHIM components are probed by the variety in properties of GRG lobes that are embedded in it. However, although GRG lobes are likely to be among the oldest and hence most relaxed they may not be in pressure balance with the ambient medium (e.g. B0503-286; Subrahmanyan et al, 2008). It then points to the strong potential of the low frequency radio surveys such as the Low-Frequency Array for Radio astronomy (LOFAR) and Murchison Widefield Array (MWA) and Square Kilometre Array (SKA) in discovering faint, relic giant radio galaxies which would be valuable in measuring directly the WHIM properties.

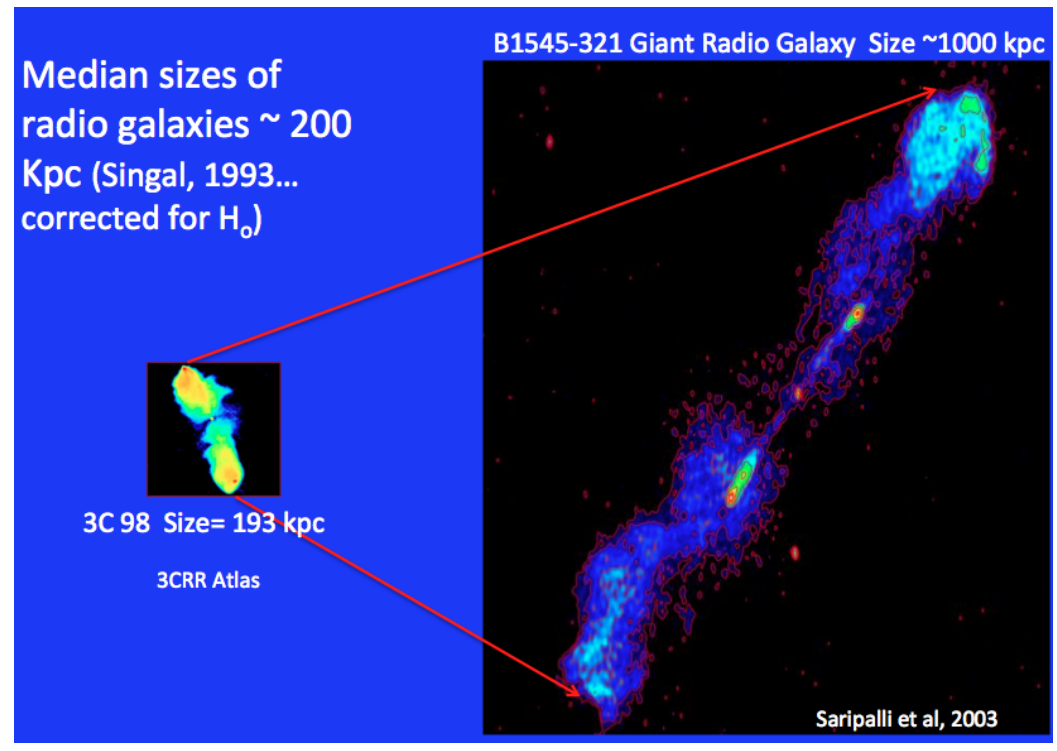

Figure 1: A comparison of sizes: Giant radio galaxy B1545-321 and the average size radio galaxy, 3C98.

For the 12 GRGs for which adequate radio continuum data was not available we imaged them at $1.4 \mathrm{GHz}$ with the Australia Telescope Compact Array in total and polarized intensity. For 17 GRGs we obtained optical spectroscopic data of the galaxy environment over a 2-deg field in the vicinity of their host galaxies using the AAOmega spectrograph on the Anglo Australian Telescope. Optical spectra were obtained for 24742 galaxies and redshifts measured for 9080 galaxies. The redshift completeness of our data is high: within $0.8 \mathrm{deg}(=2.2$ to $7.5 \mathrm{Mpc})$ of the GRG hosts it approaches 100 per cent in most cases. For the exercise only galaxies within 0.8 deg radius of the GRG host have been considered. Completeness (fraction of spectroscopically confirmed objects, both stars and galaxies, with respect to potentially observable targets) as a function of both radius and apparent magnitude, is presented in Figure 2. With the goal of relating large-scale galaxy environment around a GRG with its radio structure we used five parameters $\left(a_{1}, a_{2}, a_{3}, a_{4}, a_{5}\right)$, each of which sums up galaxies weighted by angular separation with respect to the long axis of the GRG using Fourier Transform-type components, $1, \sin (\theta), \cos (\theta), \sin (2 \theta), \cos (2 \theta)$ respectively and normalized by the mean of galaxy counts in similar volumes placed randomly in the field. Each parameter represents galaxy overdensity in regions around the GRG host, with specific relationship with the GRG structure (see Figure 3 for a pictorial description). 

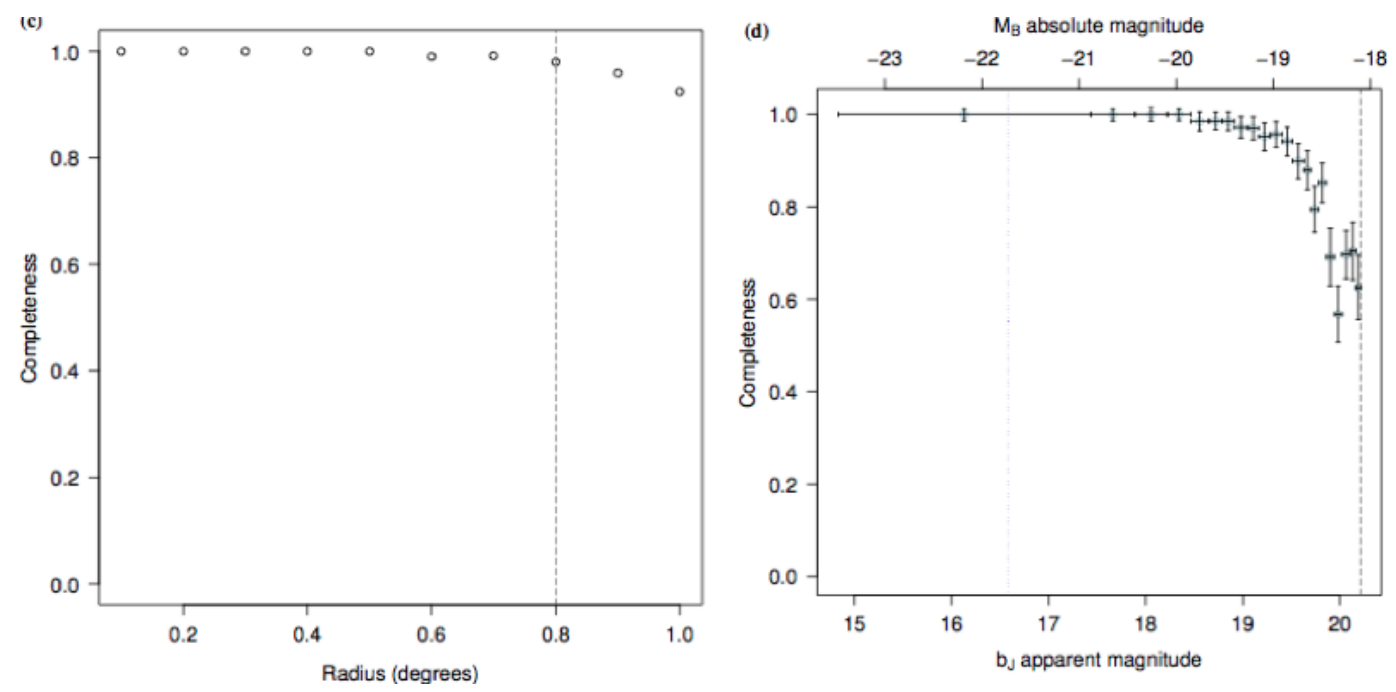

Figure 2: The two panels show completeness plots for 2-D optical spectroscopic data complete to radius 0.8-deg and absolute magnitude -19 to -20 . Figures 2-7 are from Malarecki et al, 2013 and Malarecki et al, 2015.

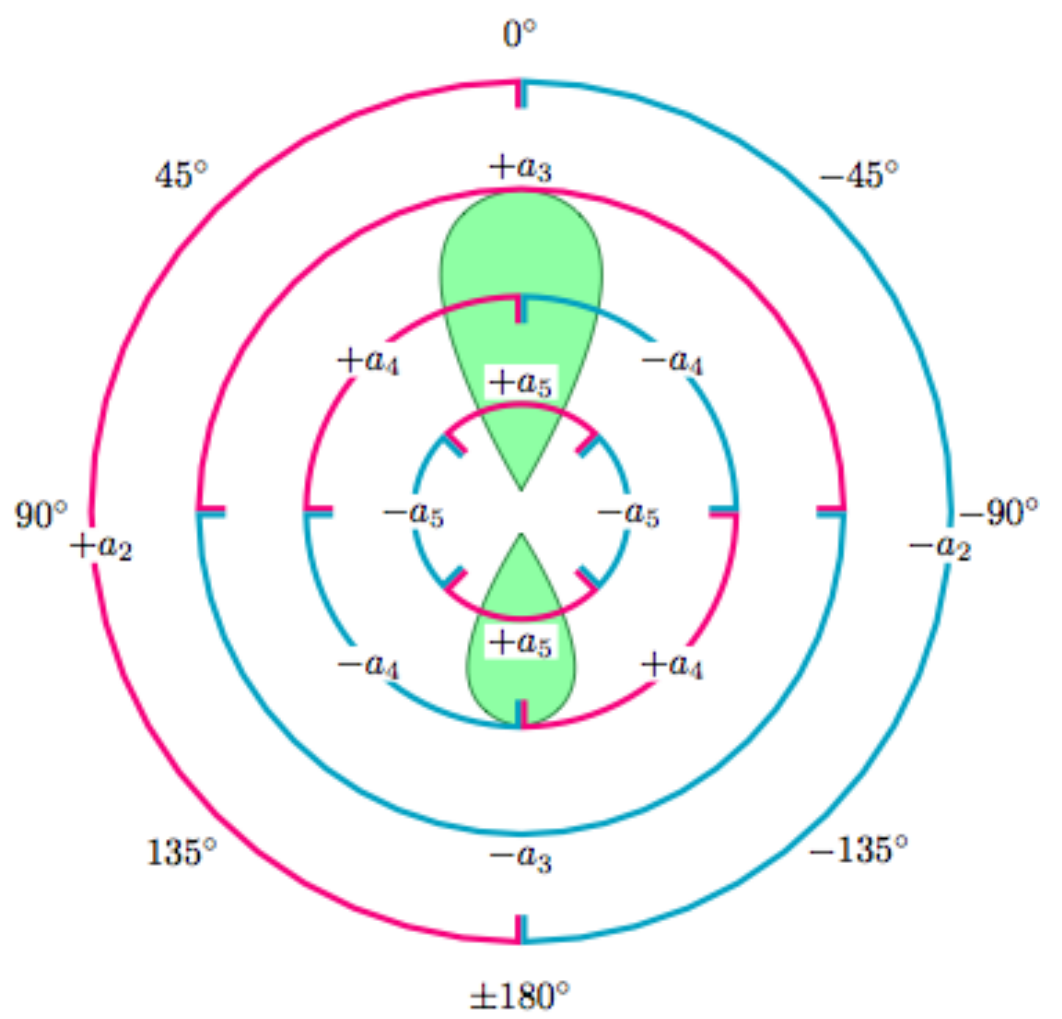

Figure 3: A sketch depicting the definition of each component of the tool used in relating galaxy environment to radio galaxy structure. The angles are defined with respect to the long axis of the GRG. 


\section{Results}

We compared lobe component pressures (estimated in locations with lowest surface brightness) with models for WHIM components from simulation studies of Schaye et al (2010). The WHIM that the GRG lobes are possibly sampling would have (1) particle overdensity in the range 50 to 500 and (2) temperature in excess of $\sim 10^{6} \mathrm{~K}$. Such temperature (density) regions would account for only 6 (1) percent of WHIM by volume or 18 (23) percent of WHIM by mass (Figure 4 and Figure 5). As mentioned above, GRG lobes may not all be in pressure balance with the ambient medium. In that case, the WHIM parameters may only be upper limits.

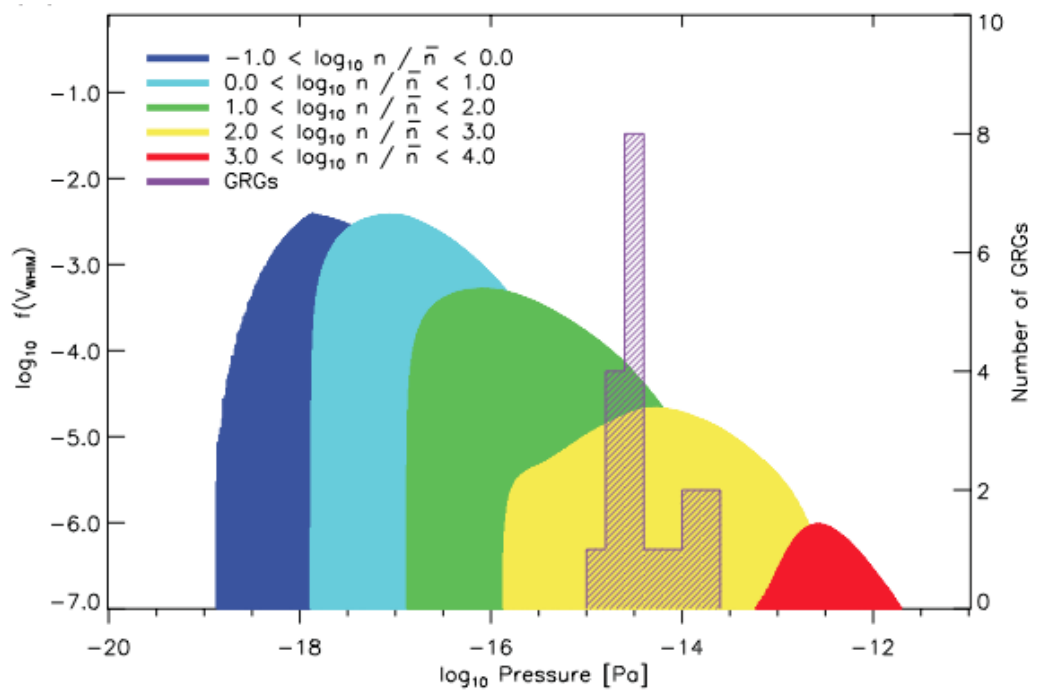

Figure 4: Probability distribution functions for the WHIM from the OverWhelmingly Large Simulations (OWLS) project (Schaye et al. 2010) as a function of pressure for different cuts in particle overdensity, overlayed with the complete sample of averaged GRG radio lobe pressures in purple.

Our estimates of the mean galaxy number overdensity in the vicinity of GRG hosts, in volumes of $700 \mathrm{Mpc}^{3}$ is $\sim 70$, indicating overdense but non-virialized environments. The attempt at relating the surrounding galaxy environment with the GRG radio structure using the Fourier componenttype analysis resulted in an outcome that $a_{3}$ is negative for asymmetric sources (or that higher galaxy over-density is seen on the side of shorter lobe) and $a_{5}$ is negative for several sources (or that there exists higher galaxy over-density in direction orthogonal to radio source axis; see section 2 and Figure 3 for description of the 'a' parameters). In addition, a strong influence of environment is seen in the form of the $a_{4}$ parameter where GRG lobes are seen to deflect away from greater galaxy over-densites (Figure 6).

On overlaying GRG radio images with the galaxy environment surrounding the GRG hosts, we find a clear influence of galaxy environment on the radio structures. GRGs appear to grow in sparse environments or perpendicular to galaxy chains and filaments. GRG jets preferentially seek void regions (Figure 7 gives an example).

We are encouraged by clear signs of interaction between galaxy distributions and GRG jets and lobes and a future study will use the exquisite data gathered on the large-scale environments around GRGs to model gas environments in GRG vicinities. 


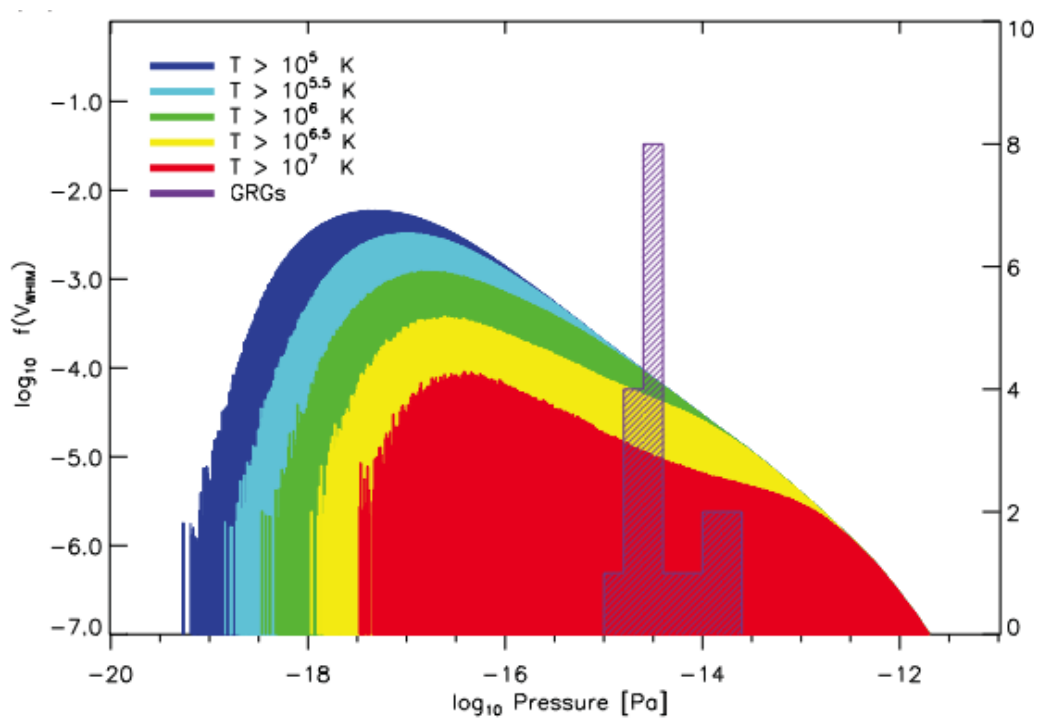

Figure 5: Probability distribution functions for the WHIM from the OWLS project (Schaye et al. 2010) as a function of pressure for different cuts in temperature, overlayed with the complete sample of averaged GRG radio lobe pressures in purple.

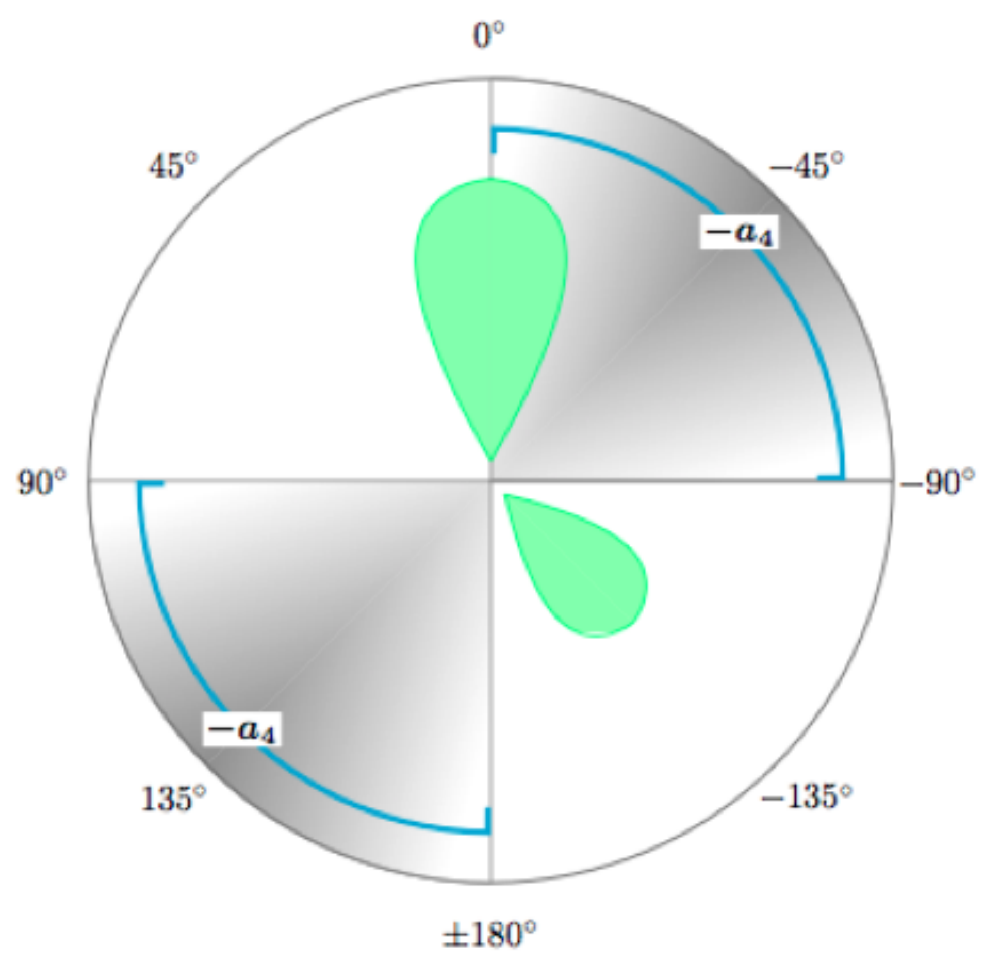

Figure 6: Sketch showing strong quadrupole moment, $a_{4}$, in the stacked galaxy distribution around GRGs with non-collinear lobes. See section 2 and Figure 3 for description of the 'a' parameters 


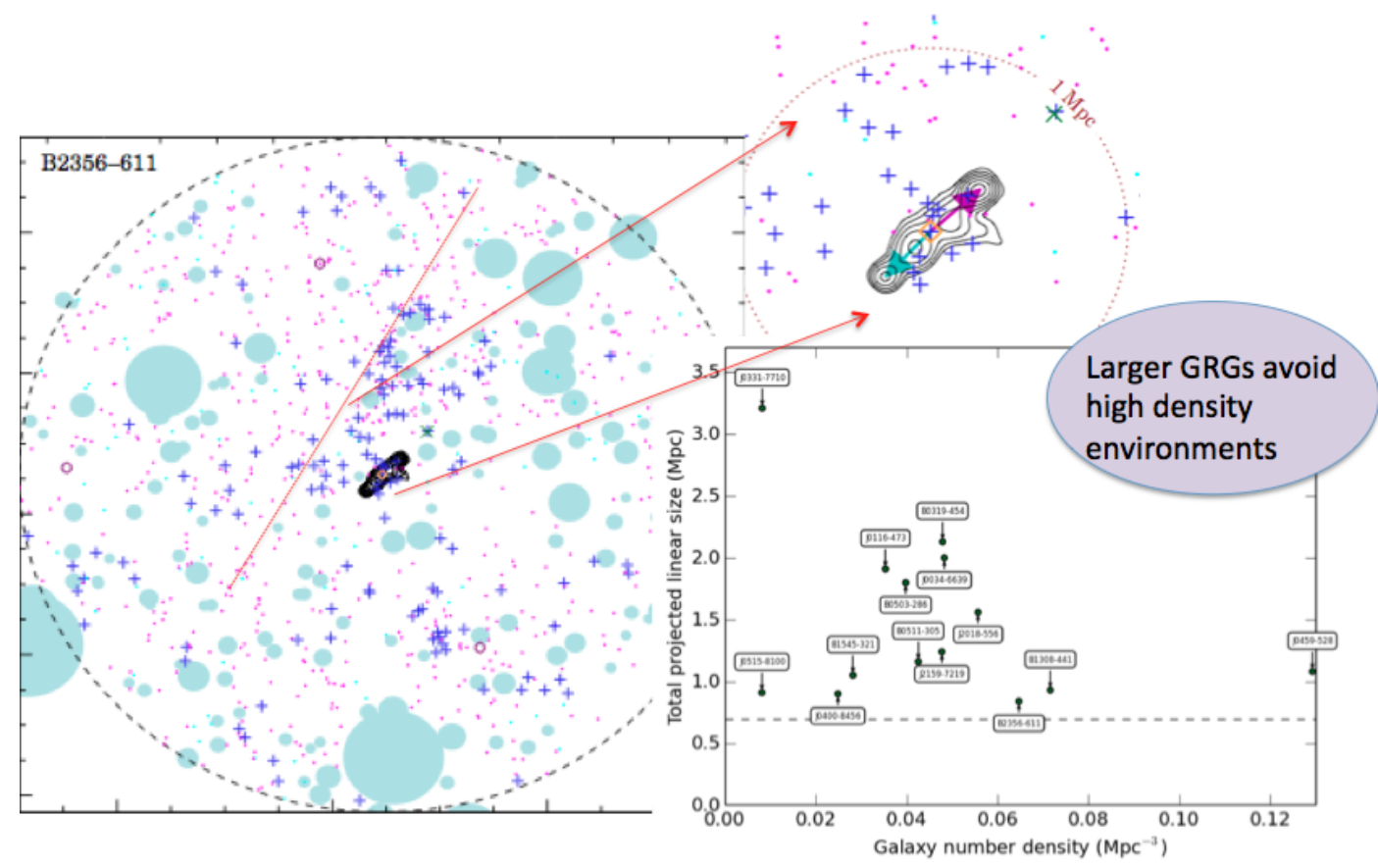

Figure 7: Large-scale galaxy distribution around B2356-611. Inset shows galaxy distribution within $1 \mathrm{Mpc}$ of the GRG host. Plot shows distribution of GRG linear sizes as a function of ambient galaxy number density.

\section{References}

[1] S. Cantalupo et al., 2014, Nature, 506, 63.

[2] R. Cen and J.P. Ostriker, 1999, ApJ, 514, 1.

[3] A. Fraser-McKelvie et al. 2011, MNRAS, 415, 1961.

[4] J.M. Malarecki et al., 2013, MNRAS, 432, 200.

[5] J.M. Malarecki et al., 2015, MNRAS, 449, 955.

[6] D.C. Martin et al., 2014, ApJ, 786, 106

[7] D.C. Martin et al., 2015, Nature, 524, 192.

[8] A. Popping and R. Braun, 2011, AA, 527, 90.

[9] V. Safouris et al., 2015, MNRAS, 393, 2.

[10] L. Saripalli et al., 2003, ApJ, 590, 181

[11] J. Schaye et al., MNRAS, 402, 1536.

[12] M.J. Shull et al., ApJ, 759, 23

[13] A.K. Singal, 1993, MNRAS, 263, 139.

[14] R. Subrahmanyan et al., ApJ, 677, 63. 